\title{
Small-Angle X-ray Scattering (SAXS) Study on Nonionic Fluorinated Micelles in Aqueous System
}

Lok Kumar Shrestha ${ }^{1}$, Suraj Chandra Sharma ${ }^{1,2}$, Takaaki Sato ${ }^{3}$, Otto Glatter ${ }^{4}$, and Kenji Aramaki $^{1^{*}}$

1. Graduate School of Environment and Information Sciences, Yokohama National University, Tokiwadai 79-7, Hodogaya-ku, Yokohama 240-8501, Japan.

2. Department of Pure and Applied Chemistry, Faculty of Science and Technology, Tokyo University of Science, 2641 Yamazaki, Noda Chiba 278-8510, Japan.

3. Division of Physics and Applied Physics, Faculty of Science \& Engineering, Waseda University, Okubo 3-4-1, Shinjuku-ku, Tokyo 169-8555, Japan.

4. Institut für Chemie, Universität Graz, Heinrichstrasse 28, A-8010 Graz, Austria.

* To whom correspondence should be addressed

E-mail: aramakik@ynu.ac.jp

Phone \& Fax: +81-45-339-4300 


\section{Abstract}

We have investigated the self-organization structures of perfluoroalkyl sulfonamide ethoxylate, $\mathrm{C}_{8} \mathrm{~F}_{17} \mathrm{SO}_{2} \mathrm{~N}\left(\mathrm{C}_{3} \mathrm{H}_{7}\right)\left(\mathrm{CH}_{2} \mathrm{CH}_{2} \mathrm{O}\right)_{10} \mathrm{H}$, a nonionic fluorinated surfactant in aqueous system by small-angle X-ray scattering (SAXS) technique. Structural modulation of the nonionic fluorinated micelle induced by temperature change, surfactant concentration, and the added fluorinated oils have been systematically studied. The SAXS data were analyzed by the indirect Fourier transformation (IFT), and the generalized indirect Fourier transformation (GIFT) depending on the volume fraction of the surfactant. Various plausible classical model calculations have been performed to confirm the consistency of the GIFT analysis of the SAXS data. Upon successive increase in temperature, the cylindrical micelles formed at lower temperatures undergo a continuous one-dimensional growth and ultimately near the cloud point an indication of flat planar like structural pattern is observed. The evolution in structure of particle near the demixing temperature may be due to onset of attractive interactions. The shape and size of the micelle is apparently unaffected by changing the surfactant concentration from 1 to $5 \mathrm{wt} \%$ at $25^{\circ} \mathrm{C}$. Nevertheless, addition of small amount of perfluoropolyether (PFPE) oil, of structure $\mathrm{F}-\left(\mathrm{CF}_{2} \mathrm{CF}_{2} \mathrm{CF}_{2} \mathrm{O}\right) n-\mathrm{CF}_{2} \mathrm{CF}_{2} \mathrm{COOH}$ $(n \sim 21)$ modulate the micellar shape and size. Long cylindrical micelles eventually transform into globular like particles. The onset cylinder-to-sphere transition in the structure of micelles in the surfactant/water/oil system is probably due to amphiphilic nature of the oil, which tends to increase the spontaneous curvature. The lipophilic part of the oil tends to reside in the micellar core, whereas, the hydrophilic part goes close to the polar head group of the surfactant so that effective cross-sectional area per surfactant molecules increases and as a result spherical micelles tend to form. 
Perfluorodecalin (PFD) also decreases size of the micelles but its effect is poor compared to the PFPE oil.

Kew Words: Fluorinated surfactants; fluorinated oils; small-angle X-ray scattering, cylindrical micelles, rheology, and oil solubilization. 


\section{Introduction:}

Amphiphilic molecules undergo self-aggregation and form a variety of self-organized structure both in polar and nonpolar solvents [1-8]. The self assembled structures and the detailed phase behavior of the conventional poly(oxyethylene)-type nonionic surfactant is extensively studied [9-13]. The self-organization of these surfactants is highly influenced by the poly(oxyethylene) chain length, surfactant concentration and the temperature. Perfluorosurfactants also behave in similar way as their hydrocarbon analogues. However, fluorinated surfactants have some distinct properties different from those of hydrocarbon surfactants. Unlike hydrocarbon surfactants, the perfluorosurfactants are more hydrophobic and reduce the surface tension of aqueous solution to a very low value, which is in general unattainable with hydrocarbon surfactants. Similarly, they show much lower critical micelle concentration (CMC) than hydrocarbon chain surfactants of same length do [14,15]. Fluorinated surfactants have high chemical and thermal stability due to highly electronegative fluorine atoms, which make fluorocarbon chain more rigid than a hydrocarbon chain $[16,17]$. Therefore these surfactants are useful in many practical applications where hydrocarbon surfactants tend to decompose.

It has been found that the aggregate structures, and hence, the intrinsic geometry of an individual amphiphile has a strong influence on the shape of the micelles [18]. The different shapes of the micellar aggregates can be characterized by the critical packing parameter defined as, $c p p=v / a_{o} l_{c}$, where $a_{\mathrm{o}}$ is the effective cross-sectional area of the head group and $v$ and $l_{c}$ are the volume and critical chain length of the hydrophobic chain, respectively. The cpp values for spherical, cylindrical, and lamellar particle are $\sim 1 / 3,1 / 3<c p p<1 / 2$, and $1 / 2<c p p<1$, respectively. Since the fluorocarbon chains are 
bulkier than the hydrocarbon chain, with the volume of $-\mathrm{CF}_{2}$ and $-\mathrm{CF}_{3}$ being higher than that of the $-\mathrm{CH}_{2}$ and $-\mathrm{CH}_{3}$, respectively [19], fluorinated surfactant having a very large head group is needed to form a spherical aggregate $(c p p \leq 1 / 3)$, to balance the effect of bulky fluorocarbon chain [20]. Therefore, cylindrical micelles are often observed at solution conditions where spherical micelles are expected in hydrocarbon surfactant systems.

Like conventional nonionic surfactants such as poly(oxyethylene), perfluorosurfactants also show a rich phase behavior in aqueous solutions [21-25]. These surfactants in aqueous solution show an upper miscibility gap with a lower critical point in the temperature-composition diagram. Temperature has a major influence on the structure of nonionic micelles and the micellar size in general increases with temperature. Therefore, rod-like or cylindrical micelles are observed at higher temperatures. Near the clouding point there is a possibility of attractive interactions [26]. When hydrocarbon oil is solubilized in elongated micelles, the surfactant layer curvature would be changed to less or more positive depending on the nature of oils. The oil induced structural transition has been well described in the literature for hydrocarbon surfactant systems. However, fewer data are available for fluorinated systems [27-30].

Recently our group has investigated the rheological behavior of the perfluoroalkyl sulfonamide ethoxylate $\left(\mathrm{C}_{8} \mathrm{~F}_{17} \mathrm{EO}_{10}\right)$ in aqueous system and observed viscoelastic wormlike micelles in surfactant/water binary system [31]. In the previous study the temperature induced micellar growth was reported but well below the clouding point and the structure of the micelles close to the cloud point could not be pictured out. Similarly, Sharma et al. (from our group) have studied the ternary phase behavior of the $\mathrm{C}_{8} \mathrm{~F}_{17} \mathrm{EO}_{10} /$ water/PFPE system and briefly mentioned about the oil solubilization [32]. 
However, they did not study about the micellar structure and the transition induced by the added fluorinated oil. In the present contribution, we report the study of structure of nonionic fluorinated micelles over a wide range of temperatures (close to cloud point) based on small-angle X-ray scattering (SAXS) experiments. We will also describe the effect of surfactant concentration and added fluorinated oils on the structure of the micelles. We have analyzed the SAXS data by model independent indirect Fourier transformation (IFT) or generalized indirect Fourier transformation technique (GIFT) depending on the volume fraction of the surfactants. We have also tested different classical model to have the quantitative information on the structure parameters of the micelle.

\section{Experimental:}

2.1 Materials. The nonionic fluorinated surfactant perfluoroalkyl sulfonamide ethoxylate, $\mathrm{C}_{8} \mathrm{~F}_{17} \mathrm{SO}_{2} \mathrm{~N}\left(\mathrm{C}_{3} \mathrm{H}_{7}\right)\left(\mathrm{C}_{2} \mathrm{H}_{4} \mathrm{O}\right)_{10} \mathrm{H}$, designated as $\mathrm{C}_{8} \mathrm{~F}_{17} \mathrm{EO}_{10}$ and shown in Scheme 1, was obtained from Mitsubishi Materials (Japan). The surfactant was purified by placing it under vacuum for 7 days in order to remove volatile components until weight became constant. The perfluoropolyether oil $\left[\mathrm{F}-\left(\mathrm{CF}_{2} \mathrm{CF}_{2} \mathrm{CF}_{2} \mathrm{O}\right) n-\mathrm{CF}_{2} \mathrm{CF}_{2} \mathrm{COOH}\right]$ abbreviated as PFPE was kindly received from Daikin Industries Ltd., Japan. The number average molecular weight of PFPE is 3600, which gives $n \sim 21$. The polydispersity is 1.14. The perfluorodecalin (PFD) is 95\% pure (mixture of cis and trans) was purchased from the Aldrich Chem. Co., Ltd. Millipore water is used in all the experiments.

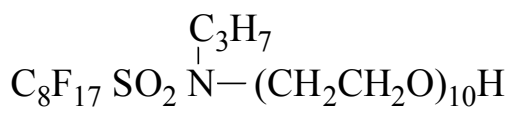


Scheme 1: Molecular structure of $\mathrm{C}_{8} \mathrm{~F}_{17} \mathrm{EO}_{10}$

2.2 Methods: 2.2.1 Sample preparations. Required amount of surfactant was taken to prepare $1 \mathrm{wt} \%$ of surfactant solution in water and taken in clean and dry glass ampoules. The samples were mixed properly by using dry thermo bath, vortex mixer and repeated centrifugation to achieve homogeneity. After mixing, the samples were placed in the temperature-controlled water bath at $25^{\circ} \mathrm{C}$ for few hours before SAXS measurements. Different samples concentrations ranges from 1 to $5 \mathrm{wt} \%$ were prepared by following the aforementioned way to study the effect of concentration on the micellar aggregates. Different concentrations of the PFPE, and PFD are added in to the $5 \mathrm{wt} \%$ surfactant solution to study the effect of added oil on the micellar structure.

2.2.2 Small-Angle X-ray Scattering (SAXS). For the structural investigation of the micellar aggregates, SAXS measurements on the dilute aqueous micellar solutions of the $\mathrm{C}_{8} \mathrm{~F}_{17} \mathrm{EO}_{10}$ were carried out. Effects of temperature, surfactant concentration and the added oils on the self-organized structure have been thoroughly examined. For the SAXS measurements, we used a SAXSess camera (Anton Paar, PANalytical) was used with a PW3830 laboratory X-ray generator with a long fine focus sealed glass X-ray tube $\left(\mathrm{K}_{\alpha}\right.$ wavelength of $\left.0.1542 \mathrm{~nm}\right)$ (PANalytical). The apparatus was operated at $40 \mathrm{kV}$ and $50 \mathrm{~mA}$. The SAXSess camera is equipped with a focusing multiplayer optics and a block collimator for an intense and monochromatic primary beam with low background, and a translucent beam stop for the measurement of an attenuated primary beam at $q=0$. Samples were enclosed into vacuum tight thin quartz capillary with an outer diameter of $1 \mathrm{~mm}$ and thickness of $10 \mu \mathrm{m}$ and the same capillary was used in each measurement to 
attain the exactly the same scattering volume and background contribution. The sample temperature was controlled with a thermostated sample holder unit (TCS 120, Anton Paar). The scattered intensities were measured with an imaging plate (IP) detection system Cyclone (Perkin Elmer, USA), and via SAXSQuant software (Anton Paar) twodimensional intensity data were transformed to the one-dimensional scattering curves as a function of the magnitude of the scattering vector. All data were normalized to the same incident primary beam intensity for the transmission calibration and were corrected for the background scattering from the capillary and the solvent. The maximum resolution of the mesurement, or $q_{\min }$, was $\sim 0.08 \mathrm{~nm}^{-1}$, which corresponds to $\sim 40 \mathrm{~nm}$ as detectable maximum size for a particle. Details on the theoretical background of the SAXS are given in supporting material.

2.2.3 Zero-Shear Viscosity. Samples for the viscosity measurements were homogenized with repeated centrifugation for 6 hours and kept in water bath at $25^{\circ} \mathrm{C}$ for 48 hours to ensure equilibration before measurements. The viscosity measurements were performed in an ARES rheometer (Rheometric Scientific) using couette (cup diameter, 17.0mm, bob diameter, $16.5 \mathrm{~mm}$, and bob length, $13.3 \mathrm{~mm}$ ) and cone-plate (diameter, $50 \mathrm{~mm}$ and cone angle $0.04 \mathrm{rad}$ ) geometries depending on samples. Temperature was controlled using a circulating fluid from a temperature-controlled water bath. A sample cover provided with the instrument was used to minimize the change in sample composition by evaporation during the measurement. The zero-shear viscosity was determined from steady shear rate measurements of samples by extrapolating the viscosity - shear-rate curve to zero shear-rate 


\section{Results and Discussions:}

\subsection{Effect of Temperature on the Micellar Growth:}

To study the effect of temperature on the geometry of the micellar aggregates, SAXS measurements were performed on $1 \mathrm{wt} \%$ surfactant solution over wide temperature ranges from 10 to $60^{\circ} \mathrm{C}$. Since system is dilute, the interparticle interaction can be assumed to be very low and neglected. Therefore, the SAXS data were analyzed by means of model independent IFT method. The normalized X-ray scattering intensity and the corresponding pair-distance distribution function (PDDF) for the $1 \mathrm{wt} \%$ surfactant solution as a function of temperature are presented in Figure 1. One can clearly see the increase of forward scattering intensity, $I(q)$, with the temperature. Absence of a correlation peak in the low- $q$ regime of the scattering functions indicates a negligible interparticle interaction. In fact, the analysis of data considering structure factor (by GIFT method) also gave identical results. The decay of $I(q) \sim q^{-1}$ in the low $q$ region suggests that the aggregates have rodlike local structure from 10 to $40^{\circ} \mathrm{C}$. However, above $40^{\circ} \mathrm{C}$, the forward intensity becomes more pronounced and follows $I(q) \sim q^{-2}$ behavior at $60^{\circ} \mathrm{C}$. In general, a growth to planar like particle should lead to this kind of behavior in the scattering curve, at least for lower concentration with negligible interparticle interaction effect $[33,34]$. The structural change with increasing temperature is more evident from the corresponding PDDF curves shown in Figure 1b. The PDDF patterns from 10 to $40^{\circ} \mathrm{C}$ exhibit a typical feature of rod-like particles as illustrated by a pronounced peak in the low- $r$ regime and an extended nearly linear tail in the high- $r$ side. Increase in temperature increases the axial length of the cylindrical micelle as indicated by the extended tail in the high- $r$ side of the PDDF curves. Upon further increasing temperature, a broad peak in the middle $r$ regime starts to develop and 
is fully developed at $60^{\circ} \mathrm{C}$, very close to the clouding temperature $\left(61^{\circ} \mathrm{C}\right)$. However, interestingly, the inflation point in the $p(r)$ curves after the maximum at low- $r$ side, which semi-quantitatively gives the cross sectional diameter of the aggregates remains practically unchanged over the wide range of temperatures. This indicates that the change in temperature could not modify the internal core structure of the micelles. Judging from the inflation point after the maximum in the $p(r)$ function the cylindrical cross sectional diameter of the rodlike aggregates is considered to be $\sim 5.8 \mathrm{~nm}$. Since the low- $q$ region in the $I(q)$ curves is proportional to the high- $r$ side of the PDDF curve, the increasing tendency of the scattering functions, $I(q)$ with temperature is the indication of the increasing $D_{\max }$ in the PDDF curve. The maximum length of the aggregate $\left(D_{\max }\right)$, depends on the maximum of resolution (or $q_{\min }$ ) of the measurement, therefore, the $D_{\max }$ as determined by the IFT method shown in the $p(r)$ functions may not be the actual length of the micelles, because we miss the scattering behavior below $q=0.08 \mathrm{~nm}^{-1}$. Nevertheless, the trends in the variation of $D_{\max }$ values as obtained from SAXS measurements can be taken as the evidence of micellar growth.

\section{Insert Figure 1}

Appearance of a bump in the scattering intensity at $50^{\circ} \mathrm{C}$ and its growing tendency with further increasing temperature can be taken as a strong evidence of the two-dimensional micellar growth. Minute observation of the PDDF curves below $40^{\circ} \mathrm{C}$ reveals a decreasing trend in the $p(r)$ functions immediately after the maximum. However, no such phenomena are observed above $50^{\circ} \mathrm{C}$, see Figure 1c. A cylinder-to-planar type of transition leads to this kind of behavior. Besides, the oscillatory-shear rheological 
behavior of the $\mathrm{C}_{8} \mathrm{~F}_{17} \mathrm{EO}_{10}$ solutions have shown that with increasing temperature, the relaxation time $\tau_{\mathrm{R}}$ (at lower concentration below $10 \mathrm{wt} \%$ ) first increases swiftly reaches maximum and then decreases [31]. With increasing concentration the position of the maximum of the $\tau_{\mathrm{R}}$ decreases. However, the value of the $\tau_{\mathrm{R}}$ is almost independent of the surfactant concentration at higher temperatures (above $40^{\circ} \mathrm{C}$ ). The decreasing relaxation time with temperature may not be due to the shortening of the micelles because the SAXS data have shown the clear evidence of increase of the micellar length with increasing temperature. Moreover, the rheological parameter $G_{\mathrm{o}}$ estimated from the Maxwell equation found to increase with temperature [31]. Thus, the possible reason for the faster relaxation at higher temperature may be due to the micellar branching i.e. formation of structures with low curvature.

However, we cannot neglect the possibility of the attractive interaction of the particles as we are very close to the demixing temperature $\left(61^{\circ} \mathrm{C}\right)$. Glatter et al. [26] have studied the structure of nonionic micelles near the critical point and found enormous micellar growth near the critical point due to strong attractive interactions. The present situation is very close to what was observed by Glatter in the past. Therefore, the evolution in structure of particle near the demixing temperature may be due to onset of strong attractive interactions. Note that what we measure is a system of particles, so this can be a cluster of cylinders, for example, we cannot exclude the possibility of existence of branched cylinders with a flat overall structure. This cannot be concluded from SAXS data and could be verified via other techniques such as Cryo-TEM.

Formation of spherical, cylindrical or planar fluorinated micelles above CMC has been reported in the past $[35,36]$ but the detail study on the temperature induced micellar 
growth and onset of critical fluctuation near the cloud points in the nonionic fluorinated surfactant systems is less studied so far. Recently, Eastoe et al. [37] have shown the formation of cylindrical or planar nonionic fluorinated micelles depending on the number and position of hydrophilic head group per surfactant molecule by small-angle neutron scattering (SANS) study. As for example, the Y-shape fluorinated surfactant $\left(\mathrm{CF}_{3}\left(\mathrm{CF}_{2}\right)_{5}\left(\mathrm{CH}_{2}\right)_{2} \mathrm{~N}\left[\left(\mathrm{CH}_{2} \mathrm{CH}_{2} \mathrm{O}\right)_{3} \mathrm{H}\right]_{2}\right)$, where both head groups are at the same end of the hydrophobic group presents cylindrical micelles with average length of $\sim 12 \mathrm{~nm}$. On the other hand, linear monodisperse fluorinated block copolymer EO-CF-EO, (bolaform type) presents lamellar micelles. The HLB number of the present surfactant $\mathrm{C}_{8} \mathrm{~F}_{17} \mathrm{EO}_{10}$ and the Y-shaped surfactant are calculated to 8.9 and 8.4, respectively. Although, the HLB number of the $\mathrm{C}_{8} \mathrm{~F}_{17} \mathrm{EO}_{10}$ is slightly higher than the $\mathrm{Y}$-shaped surfactant, it forms longer cylindrical micelles $(\sim 32 \mathrm{~nm})$ compared to the $\mathrm{Y}$-shaped surfactant. This discrepancy is attributed to the bulky head group of the Y-shaped surfactant. Since both the hydrophilic head groups are present at the same end of the hydrophobic group in the Y-shaped surfactant, the effective cross-sectional area per surfactant molecule could be larger than that of the $\mathrm{C}_{8} \mathrm{~F}_{17} \mathrm{EO}_{10}$ system and tends to form less elongated structure due to smaller critical packing parameter.

3.2 Effects of Surfactant Concentration on the Micellar Structure: In order to study the effect of surfactant concentration on the micellar structure we have performed a series of SAXS measurements on 1 to $5 \mathrm{wt} \%$ surfactant solutions, which are well above the CMC $(0.005 \mathrm{mM})[38]$. In Figure 2, we present the normalized scattering functions $I(q)$ and the corresponding PDDF, $p(r)$ functions extracted from the GIFT analysis of the SAXS data depending on the surfactant concentration at fixed temperature $25^{\circ} \mathrm{C}$. For the sake of 
clarity, the data for $1 \mathrm{wt} \%$ surfactant is also included in the Figure 2. For the dense systems, we cannot neglect the effect of interparticle interaction. Therefore, the SAXS data above $2 \mathrm{wt} \%$ surfactant were analyzed by GIFT method.

The structure of the micelles largely depends on the nature of the surfactants. Most of the surfactants with bulky head group tend to form small spherical micelles above CMC. In general, such micelles grow with increasing the volume fraction of the surfactant and hence they increase in the aggregation number. Such growth is well explained by the concept of thermodynamics and is attributed to the entropy effect. In contrast to the hydrocarbon analogous, the fluorocarbon surfactant perfluoroalkyl sulfonamide ethoxylate forms cylindrical micelles above CMC due to strongly associated hydrophobic fluorocarbon chains and small head groups. In the present system we have observed that the surfactant concentration could not modulate the structure of the particles at least within the studied concentration limit. With increasing surfactant concentration from 1 to $5 \mathrm{wt} \%$ no significant changes in the maximum length of the micelle is observed, see Figure $2 \mathrm{~b}$. The phase behavior of the $\mathrm{C}_{8} \mathrm{~F}_{17} \mathrm{EO}_{10}$ /water system showed that with increasing surfactant concentration the micellar phase separates to hexagonal or lamellar liquid crystal phase depending on the temperature [31]. At lower temperatures below $\left(15^{\circ} \mathrm{C}\right)$ the micellar phase separates to hexagonal liquid crystal and at higher temperatures to lamellar liquid crystal phase. At $25^{\circ} \mathrm{C}$, the lamellar liquid crystal phase is detected beyond $60 \mathrm{wt} \%$. Thus, it is possible to observe planar like micelles at very high surfactant concentration close to the lamellar liquid crystal phase. However, within the studied range of concentrations, we did not observe any signature of the planar like micelles at $25^{\circ} \mathrm{C}$. As shown in Figure $2 \mathrm{~b}$, all the PDDF curves exhibit the typical features of elongated cylindrical micelles as anticipated by a pronounced 
peak in the low- $r$ side and an elongated tail to the higher- $r$ side. The maximum length of the micelles, $D_{\max }$, as indicated by down arrow is virtually constant within the studied surfactant concentration limit. Besides, the position of inflection point (as indicated by broken line in Figure 2b) seen on the higher- $r$ side of the maximum of the PDDF curves is unchanged at all volume fractions of the surfactants. This confirms that the local internal structure that is the cross-sectional diameter of the micelles remains unchanged and unaffected by the change in the surfactant concentration.

\section{Insert Figure 2}

The continuous increase of the scattering intensity with surfactant concentration throughout the entire $q$ range, and hence, the $p(r)$ function in the low- $r$ side is due to the increase in the number density of the scattering particles in the unit scattering volume. Minute observation of the scattering functions reveals that with increasing surfactant concentration, the forward scattering intensity becomes suppressed, and leads to the formation of a weak but growing interaction peak at intermediate $q$ values. This growing peak indicates the strong repulsive intermicellar interaction owing to the decreased osmotic compressibility of the system at higher surfactant concentration $[39,40]$.

3.3 Effects of Oils on the Micellar Structure: Various nonpolar oils can be solubilized in the micellar core due to its oily environment. These oils influence the spontaneous curvature of the self-organized structure and modulate the size, shape, and aggregation number of the micelles. The impact on the curvature and hence degree of structural modulation depends on the residence of the oils in the surfactant aggregates. Two 
extreme cases of complete swelling or complete penetration is well accepted $[41,42]$. Thus the oils, depending on its nature, either goes to the hydrophilic/lipophilic interface of the surfactant molecule or at the micellar core or in between the hydrophobic chains and accordingly modifies the geometry of the micelle. The solubilization of fluorinated oils in fluorinated surfactant/water system has been studied [43] but the details on the structure of micelles induced by the added oils is not well studied. In the present investigation, we the structural modification of the nonionic fluorinated micelles induced by the solubilized oils.

The PFPE and PFD are immiscible with water at room temperature but they are soluble in the micellar solution of the $\mathrm{C}_{8} \mathrm{~F}_{17} \mathrm{EO}_{10}$. Recently published ternary phase diagram of the $\mathrm{C}_{8} \mathrm{~F}_{17} \mathrm{EO}_{10}$ /water/PFPE system has shown that the oil solubilization increases with the $\mathrm{C}_{8} \mathrm{~F}_{17} \mathrm{EO}_{10}$ concentration and after reaching a maximum finally decreases. The ternary phase diagram gives a clear picture on the formation of microemulsion with about $28 \mathrm{wt} \%$ of the PFPE solubilized at the interior of the micelles at $45 \mathrm{wt} \%$ surfactant. However, in the dilute region, say at $5 \mathrm{wt} \%$ surfactant only about $1.5 \mathrm{wt} \%$ oil can be solubilized [32] so that formation of microemulsion cannot be confirmed. In the present study, we have investigated how the solubilized fluorinated oils (perfluoropolyether, PFPE and perfluorodecalin, PFD) modulate the structure of the $\mathrm{C}_{8} \mathrm{~F}_{17} \mathrm{EO}_{10}$ micelles by the SAXS technique. Both the oils induced cylinder-to-sphere transition on the micellar structure. In Figure 3, we present the scattering functions and the corresponding PDDF curves for the mixed surfactant/water/PFPE system as a function of PFPE concentration at $25^{\circ} \mathrm{C}$.

\section{Insert Figure 3}


As can be seen from the scattering data, the forward scattering intensity is decreasing with increasing the amount of PFPE. A $q^{-1}$ behavior in the low- $q$ observed in the system without the addition of oil, changes to the $\sim q^{0}$ behavior when the PFPE concentration in the total system is reached to $0.5 \mathrm{wt} \%$. This type of behavior in the scattering curves indicates the cylinder-to-sphere transition in the geometry of the aggregates. The structural transition of the micelle is clearer in the PDDF curves shown in Figure $3 \mathrm{~b}$. The amphiphilic oil PFPE monotonously decreases the maximum length of the micelles as indicated by the down arrow in the Figure $3 \mathrm{~b}$. The long cylindrical particle with a maximum length of $\sim 30 \mathrm{~nm}$ transformed to globular like particle of size $\sim 10 \mathrm{~nm}$ by the addition of the $0.5 \mathrm{wt} \%$ PFPE. Nevertheless, the rod-like shape of the particles is retained keeping cross section diameter practically unchanged at 5.8nm until the PFPE concentration is reached to $0.3 \mathrm{wt} \%$. In the light of these results, we can assume that the polar part of the PFPE goes close to the hydrophilic/lipophilic interface of the surfactant molecule and thus by increasing the effective head group size, increases the interfacial curvature. However, we cannot exclude the possibility of formation of small oil pool in the micellar core. This is because the hydrophobic chain of the polymeric oil is longer than that of the host surfactant molecule. The present situation is somewhat similar to the report by Kaneko et al [44]. They reported the formation of hydrophobic oil pool in the micellar core upon adding silicon copolymer oils with longer hydrophobic part compared to the host surfactant molecule.

Next, we present the structural changes brought by the addition of PFD in the surfactant/water system at $25^{\circ} \mathrm{C}$. The decreasing tendency of the micellar size with PFD concentration can be clearly seen in the scattering intensity and the PDDF curves 
displayed in Figure 4. The decrease in the forward scattering intensity and of the maximum micellar length $D_{\max }$ with increasing PFD is the signature of the shrinkage. When $0.3 \% \mathrm{PFD}$ is introduced in the $5 \mathrm{wt} \%$ surfactant solution, the size of the long cylindrical micelles $D_{\max } \sim 30 \mathrm{~nm}$ reduced to $\sim 17 \mathrm{~nm}$. However, the rod-like shape of the micelles is retained. Features of rod-like micelles can still be seen in the $p(r)$ functions with a pronounced peak in the low- $r$ region and an extended tail in the high- $r$ side. Thus, in contrast to the PFPE, no modulation in the shape of the micelles is induced by hydrophobic PFD oil within the studied concentration limit. However, minute observation of the PDDF curves reveals a slight increase of the cross-section diameter of the micelles. The inflection point after the maximum, which semi quantitatively gives the cross-section diameter of the micelle increases, but slightly with PFD, see Figure $4 \mathrm{~b}$. Such change in the internal structure of the micelle is possibly due to the strongly hydrophobic nature of the oil PFD. Therefore, the PFD tends to form an oil pool in the micellar core. The increasing tendency of the cross-section diameter in the PFD system thus can be attributed to the formation of small oil pool swelling the micelle. Nevertheless, in spite of the cyclic structure of the PFD, it is possible that it may penetrate the fluorocarbon chain of the surfactant and tend to go near to the interface and thus by increasing spontaneous curvature reduces the size of the particles.

\section{Insert Figure 4}

3.4 Micellar Structure Versus Viscosity: It is well known that the structure of the particle has a major influence on the rheology and hence on the viscosity of the solution. Depending on the geometry of the particles, the viscosity of the solution differs widely. 
Compared to the short globular micellar solution, the solution of longer cylindrical micelles exhibits higher viscosity. At higher concentration, these cylindrical micelles entangle to form a rigid network of viscoelastic wormlike micelles. In the present study, we have performed steady-shear rheological measurements on the series of samples containing different amount of fluorinated oils (PFPE and PFD). The zero-shear viscosity $\left(\eta_{o}\right)$ was calculated by fitting the experimental data to Carreau model. The plot of $\eta_{o}$ versus oils concentration at fixed surfactant concentration and the corresponding length of micelles extracted by the GIFT analysis of the SAXS data are presented in Figure 5.

\section{Insert Figure 5}

The Figure 5a shows that the viscosity of $5 \mathrm{wt} \%$ surfactant solution is pretty high compared to that of the pure water. Such a high viscosity (20Pa.s) of the solution is attributed to the formation of long cylindrical micelles. However, the addition of small amount of the fluorinated oils (PFPE or PFD) dramatically reduces the viscosity of the solution, which is may be due to cylinder-to-sphere type of transition in the micellar structure. The SAXS measurements have shown that the addition of oils modulates the structure of the micelles in terms of shape and size. The addition of the PFPE and PFD induces cylinder-to-sphere transition and the length of the micelle decreases monotonously with the oils concentration in

the surfactant/water/oil systems, see Figure 5b. Addition of the PFPE oil induces a monotonous decrease in the viscosity of the solution and the viscosity decreased by four orders of magnitude upon addition of the $1 \mathrm{wt} \%$ PFPE. On the other hand, addition of the PFD could 
not decrease the viscosity until a minimum concentration is reached, although SAXS data have shown the shortening of the micelles. This discrepancy might be due to higher degree of

micellar entanglement in the PFD system. Besides, it has been observed that the viscosity of the PFD system is higher throughout the concentration regime studied compared to the PFPE system. This highlights the poor ability of the PFD for the cylinder-to-sphere transition and in fact this is what we have observed from the SAXS. Of course, the PFD shortens the length of micelles but the rod-like features are still retained by the system. Thus, the rheological behavior (decrease of $\eta_{o}$ with oil concentration) is strongly supported by the SAXS data.

\subsection{Internal Structure of the Micelles:}

We have calculated $p_{\mathrm{c}}(r)$ corresponding to Equation (6), see supporting information and $\Delta \rho_{\mathrm{c}}(r)$ for the surfactant/water and surfactant/water/oil systems at $25^{\circ} \mathrm{C}$ and the results are presented in Figure 6. The model-independent approach provides the cross-section diameter of $\sim 5.8 \mathrm{~nm}$ as estimated from the maximum dimension, $D_{\mathrm{c} \max }$, of the $p_{\mathrm{c}}(r)$ for the surfactant/water system. This is quantitatively complementary with the estimation made from the total $p(r)$ described in the previous section. The maximum cross section radius, $R_{\mathrm{c} \max }$, from the $\Delta \rho_{\mathrm{c}}(r)$ showing $\sim 2.9$ $\mathrm{nm}$ further confirm it.

\section{Insert Figure 6}

With increasing concentration of the PFPE, the $D_{c \max }$ is practically unchanged as can be seen from the cross-sectional PDDF curves displayed in Figure 6a. Whereas, in the PFD system a slight increase in the $D_{c \max }$ is observed. However, with the available data we cannot precisely say that micelles swell in the PFD system, but the results semi-quantitatively indicate that the 
added PFD tends to incorporate in the micellar core. The corresponding electron density profiles of the surfactant/water binary and the surfactant/water/oil ternary systems are presented in Figure 6b, where the arrow indicates the cross-sectional radius of the micelles. Note that the positive electron density profile is due to the fact that both the fluorocarbon chain and the hydrophilic head group of the surfactant have higher electron density compared to that of the solvent water.

3.6 Model Calculations: In the present study we have used the IFT approach or its generalized version GIFT, and additionally the model-free cross section IFT analysis to investigate the structure of the micellar aggregates. These techniques give real space and specific information on the investigated system. However, a model calculation, which involves the fitting of the experimental $I(q)$ with a theoretical function calculated from structure models is a common tool to determine the detailed information on the structure of the aggregates. Therefore, as a complementary method to provide quantitative information about the structure parameter we have performed model calculation to the selected systems. The calculation was done based on the method reported [45]. On the basis of the structures derived from the GIFT analysis, we have selected different models depending on the systems. As for example, for the $1 \mathrm{wt} \%$ surfactant system we have used homogenous cylinder model and for the surfactant/water/oil systems we have used homogenous ellipsoidal prolate models. The selected models are well fitted the experimental scattering function and the results are presented in Figure 7. The cylindrical model estimates the cross section radius of the particles to $2.70 \mathrm{~nm}$ with maximum length of $28.5 \mathrm{~nm}$. Similarly, the short and long axes of the ellipsoidal prolate in the $5 \mathrm{wt} \%$ surfactant $+0.5 \%$ PFPE system is estimated to 2.69 and $4.60 \mathrm{~nm}$ respectively. Both the structure parameters (short and long axes) are slightly increased in the PFD system. The short 
and long axes are estimated to 2.71 and $9.24 \mathrm{~nm}$ respectively. The structure parameters derived from the model calculations are very much close to the values obtained by the GIFT analysis. In the Figure 7, the open symbols represent the experimental SAXS data and the solid lines stand for the scattering function from the model calculation.

We have successfully confirmed that all the results from the model calculation are basically consistent with those obtained with GIFT/IFT and the cross section IFT.

\section{Insert Figure 7}

\section{Conclusion:}

We have investigated the structure of the nonionic fluorinated micelles in aqueous system by means of SAXS. Both IFT and GIFT methods are used to analyze the SAXS data depending on the volume fraction of the surfactant. The results extracted form the IFT or GIFT analysis of the SAXS data show the increasing tendency of the micellar size with increasing temperature. Near the cloud point temperature, a strong increase of the scattering intensity in forward direction is observed and a cylinder-to-planar type of transition is clearly seen in the PDDF curves, which is assumed due to the onset of attractive interactions independent of the actual size or shape of the micelles. Such behavior in the fluorinated micellar structure is poorly reported so far. Increasing surfactant concentration from 1 to $5 \mathrm{wt} \%$ could not induce any changes in the maximum length of the micelles, although the micellar phase transforms into lamellar liquid crystal phase at higher surfactant concentration $(\sim 60 \mathrm{wt} \%)$ at $25^{\circ} \mathrm{C}$. Thus, it is possible to speculate that the cylindrical micelles are in equilibrium with the planar like micelles at higher surfactant concentration close to the lamellar liquid crystal phase. 
Solubilization of fluorinated oils in the nonionic or ionic fluorinated micelles and formation of microemulsion is studied. However, the structural transition in the micellar structure induced by the added oil is not well studied. In the present investigation, we found that addition of amount of fluorinated oils, such as PFPE and PFD could induce a dramatic change in the structure of the $\mathrm{C}_{8} \mathrm{~F}_{17} \mathrm{EO}_{10}$ micelles, which is in general unattainable by the hydrocarbon oils. Such properties enhance the importance of the fluorinated oils from application viewpoint such as in the synthesis of nanoparticles or mesoporous materials. Due to structural transition of the micellar structure the rheological properties of the surfactant/water/oil systems significantly modified. The viscosity of the solution decreases dramatically (four orders of magnitude by incorporating $1 \mathrm{wt} \%$ PFPE). Thus, the present investigation highlights the possible routes to the structural control of the fluorinated micelles by tuning temperature, surfactant concentration, and added fluorinated oils. We believe that the present study advances the field about the structure, properties, and solubilization in fluorocarbon surfactant micelles.

\section{Acknowledgement:}

L. K. Shrestha is thankful to the Ministry of Education, Culture, Sports, Science and Technology (MEXT) of Japan for the Monbukagakusho Scholarship. This work was

partly supported by Core Research for Evolution Science and Technology (CREST) of JST Corporation.

\section{Reference:}

[1] H. Kunieda, K. Shigeta, K. Ozawa, M. Suzuki, J.Phys.Chem.B 101 (1997) 7952. 
[2] K. Aramaki, U. Olsson, Y. Yamaguchi, H. Kunieda, Langmuir 15 (1999) 6226.

[3] H. Kunieda, Shigeta K., M. Suzuki, Langmuir 15 (1999) 3118.

[4] J. Penfold, E. Staples, I. Tucker, P. Cummins, J. Colloid Interface Sci. 185 (1997) 424.

[5] C. Rodriguez, Md. H. Uddin, K. Watanabe, H. Furukawa, A. Harashima, H. Kunieda, J. Phys. Chem B 106 (2002) 22.

[6] L.K. Shrestha, M. Kaneko, T. Sato, D.P. Acharya, T. Iwanaga, H. Kunieda, Langmuir 22 (2006) 1449.

[7] L.K. Shrestha, T. Sato, D.P. Acharya, T. Iwanaga, K. Aramaki, H. Kunieda, J. Phys. Chem B 110 (2006) 12266.

[8] L.K. Shrestha, T. Sato, K. Aramaki, J. Phys. Chem B 111 (2007) 1664.

[9] H. Kunieda, H. Kabir, K. Aramaki, K. Shigeta, J. Mol. Liq. 90 (2001) 157.

[10] K. Shigeta, U. Olsson, H. Kunieda, Langmuir 17 (2001) 4717.

[11] H. Kunieda, M. Kaneko, M.A. López-Quintela, M. Tsukahara, Langmuir 20 (2004) 2164.

[12] K. Shinoda, J. Colloid Interface Sci. 34 (1970) 278.

[13] R. Strey, R. Schomäcker, D. Roux, F. Nallet, U. Olsson, J. Chem. Soc., Faraday Trans. 86 (1990) 2253.

[14] K. Shinoda, M. Hato, T. Hayashi, J. Phys. Chem. 76 (1972) 909.

[15] H. Kunieda, K. Shinoda, J. Phys. Chem. 80 (1976) 2468.

[16] T. Asakawa, M. Mouri, S. Miyagishi, Langmuir 5 (1989) 343.

[17] H. Hoffman, J. Kalus, H. Thurn, Prog. Colloid Polym. Sci. 261 (1983) 1043.

[18] Israelachvili, J. N. Mitchell, D. J. Ninham, B. W. J. Chem. Soc. Faraday Trans. 2, $72(1976) 1525$.

[19] C. Tanford, The Hydrophobic Effect: Formation of Micelles and Biological Membranes, 2nd ed.; John Wiley \& Sons: New York, 1980.

[20] C. El Moujahid, J. C. Ravey, V. Schmitt, M. J. Stébé, Colloid Surf. A 136 (1998) 289.

[21] K. Kratzat, F. Guittard, E. Taffin de Givenchy, A. Cambon, Langmuir 12 (1996) 
6346.

[22] G.J.T. Tiddy, Symp. Faraday Soc. 5 (1971) 150.

[23] G.J.T. Tiddy, B.A. Wheeler, J. Colloid Interface Sci. 47 (1974) 58.

[24] E. Everiss, G.J.T. Tiddy, B.A. Wheeler, J. Chem. Soc., Faraday Trans. 72 (1976) 1747.

[25] F. Caboi, A. Chittofrati, P. Lazzari, M. Monduzzi, Colloid \& Surf. A. 160 (1999) 47.

[26] O. Glatter, G. Fritz, H. Lindner, P. J. Brunner, R. Mittelbach, R. Strey, S. U. Egelhaaf, Langmuir 16 (2000) 8692.

[27] H. Hoffmann, W. Ulbricht, J. Colloid Interface Sci. 129, (1989), 388.

[28] C. Rodríguez, K. Aramaki, Y. Tanaka, M.A. López-Quintela, M. Ishitobi, H. Kunieda, J. Colloid Interface Sci. 291, (2005), 560.

[29] T. Sato, D.P. Acharya, M. Kaneko, K. Aramaki, Y. Singh, M. Ishitobi, H. Kunieda, J. Dispersion Sci. Technol. 27, (2006), 611.

[30] N.K. Pokhriyal, J. V. Joshi, P.S. Goyal, Colloids Surf. A 218, (2003), 201.

[31] D.P. Acharya, S.C. Sharma, C. Rodriguez-Abreu, K. Aramaki, J. Phys. Chem. B 110 (2006) 20224.

[32] S.C. Sharma, H. Kunieda, J. Esquena, C. Rodriguez-Aberu, J. Colloid and Interface Sci. 299 (2006) 297.

[33] O. Glatter, J. Appl. Cryst. 13 (1980) 577.

[34] C. Moitzi, N. Freiberger, O. Glatter, J. Phys. Chem. B 109 (2005) 16161.

[35] S. S. Beer, R.R.M. Jones, J. Phys. Chem. 93 (1989) 2555

[36] J. Eastoe, J.S. Dalton, A. Downer, G. Jones, D. Clarke, Langmuir, 14 (1998) 1937.

[37] J. Eastoe, S.E. Rogers, L.J. Martin, A. Paul, F. Guittard, E. Guittard, R.K. Heenan, J. R.P. Webster, Langmuir, 22 (2006) 2034.

[38] C. Rodriguez, Aberu, H. Kunieda, J. Dispersion Sci. Technol.26, (2005), 435

[39] A. Stradner, H. Sedgwick, F. Cardinaux, W.C.K. Poon, S.U. Egelhaaf, P. Schurtenberger, Nature 432 (2004) 492.

[40] A. Stradner, O. Glatter, P. Schurtenberger, Langmuir 16 (2000) 5354.

[41] H. Kunieda, K. Ozawa, K.-L. Huang, J. Phys. Chem. B 102 (1998) 831.

[42] H. Kunieda, M. Horii, M. Koyama, K. Sakamoto, J. Colloid Interface Sci. 236 (2001) 78 . 
[43] R. Belta, J.L. Blin, M.J. Stébé, J. Phys. Chem. B. 110 (2006) 23547.

[44] M. Kaneko, K. Matsuzawa, Md. H. Uddin, M.A. López-Quintela, H. Kunieda, J. Phys. Chem. B 108 (2004) 12736.

[45] O. Glatter, Acta Phys. Austriaca 52 (1980) 243. 

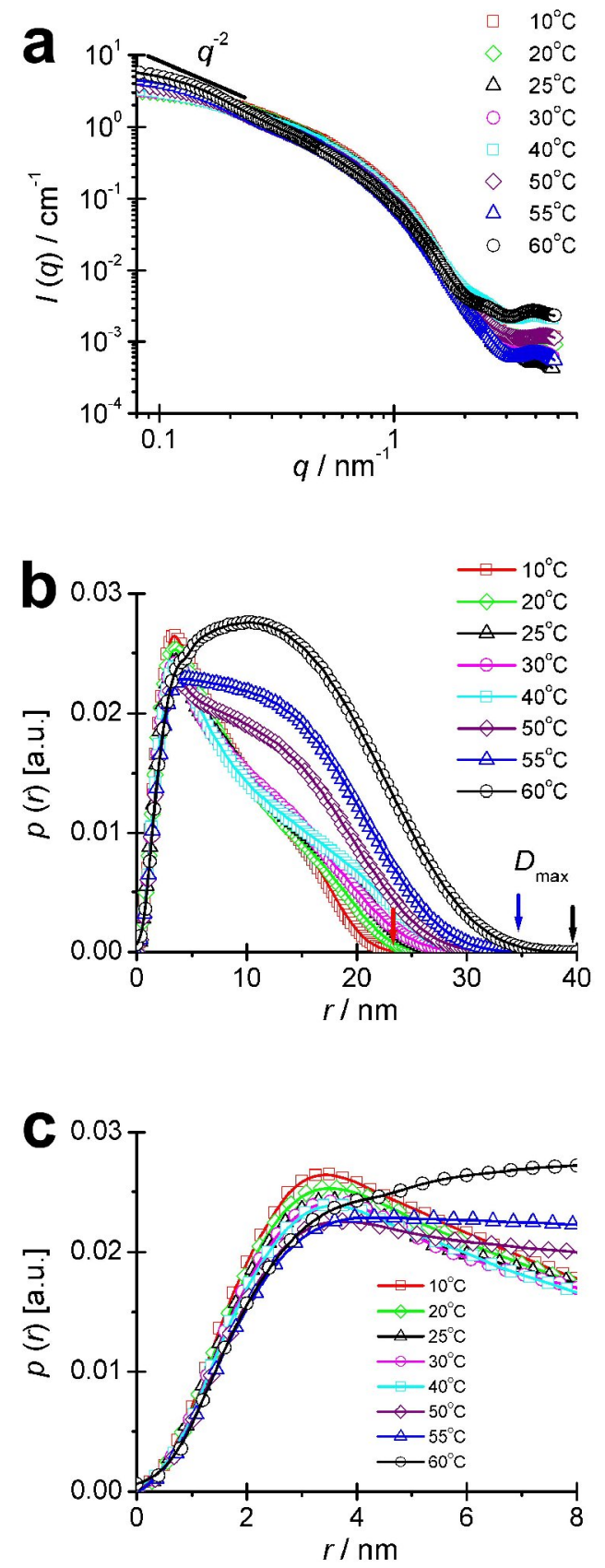

Figure 1: (a) The scattering curves $I(q)$, of the aqueous solutions of $1 \mathrm{wt} \%$ surfactant obtained in absolute unit as a function of temperature, (b) the corresponding pair-distance distribution functions (PDDF), $p(r)$, extracted by IFT analysis, and (c) the closer view of $p(r)$ functions in the low- $r$ side. The solid line in (a) represents the fit to the experimental data by IFT. The arrow in (b) indicates the maximum length of the micelles. 

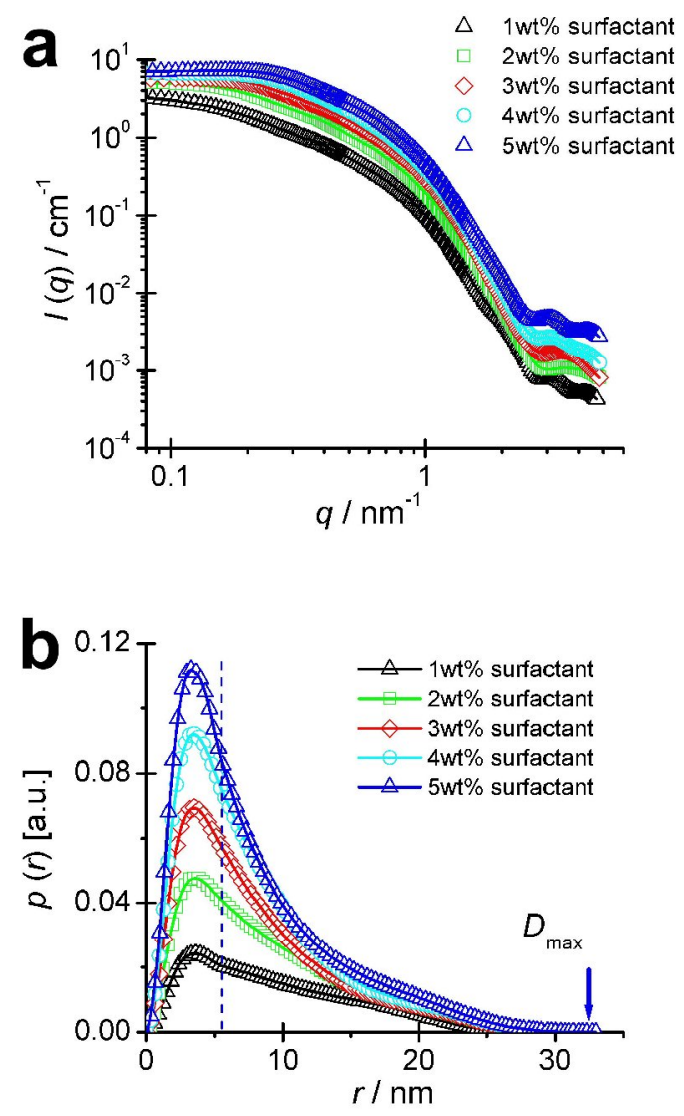

Figure 2: (a) The scattering functions $I(q)$, as a function of the surfactant concentration obtained in absolute unit at $25^{\circ} \mathrm{C}$, (b) the corresponding pair-distance distribution functions (PDDF), $p(r)$, extracted with GIFT analysis. The solid line in (a) represents the GIFT fit. The arrow in (b) indicates the maximum length of the micelle. 

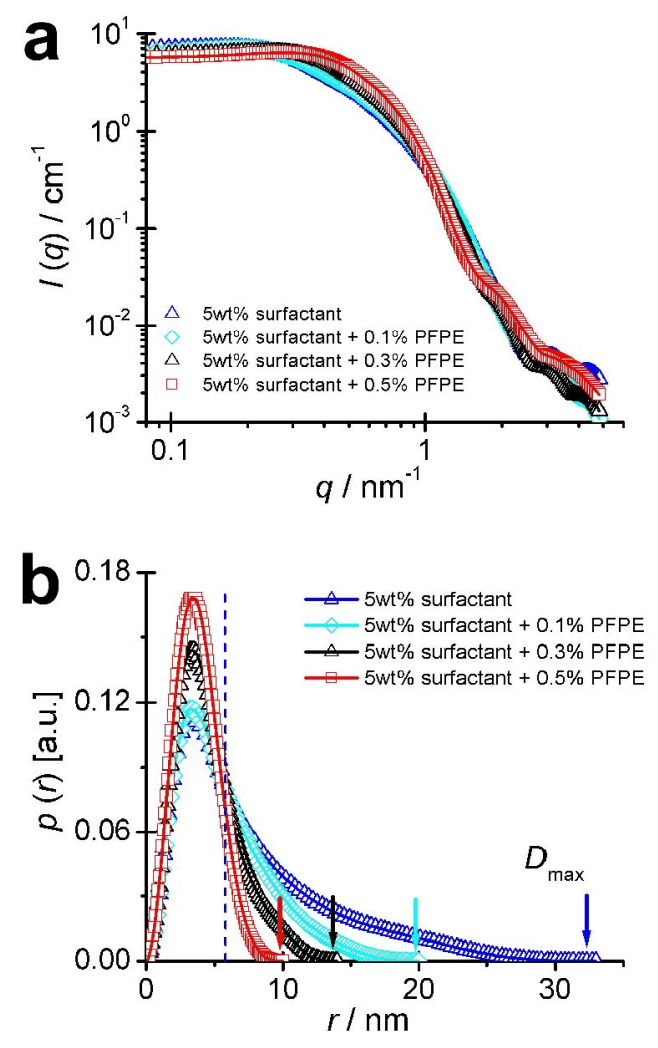

Figure 3: (a) The normalized scattering functions $I(q)$, as a function of the PFPE oil obtained in absolute unit at $25^{\circ} \mathrm{C},(\mathrm{b})$ the corresponding pair-distance distribution functions (PDDF), $p(r)$, extracted with GIFT analysis. The downward arrows in (b) represent the maximum length of the micelles. 

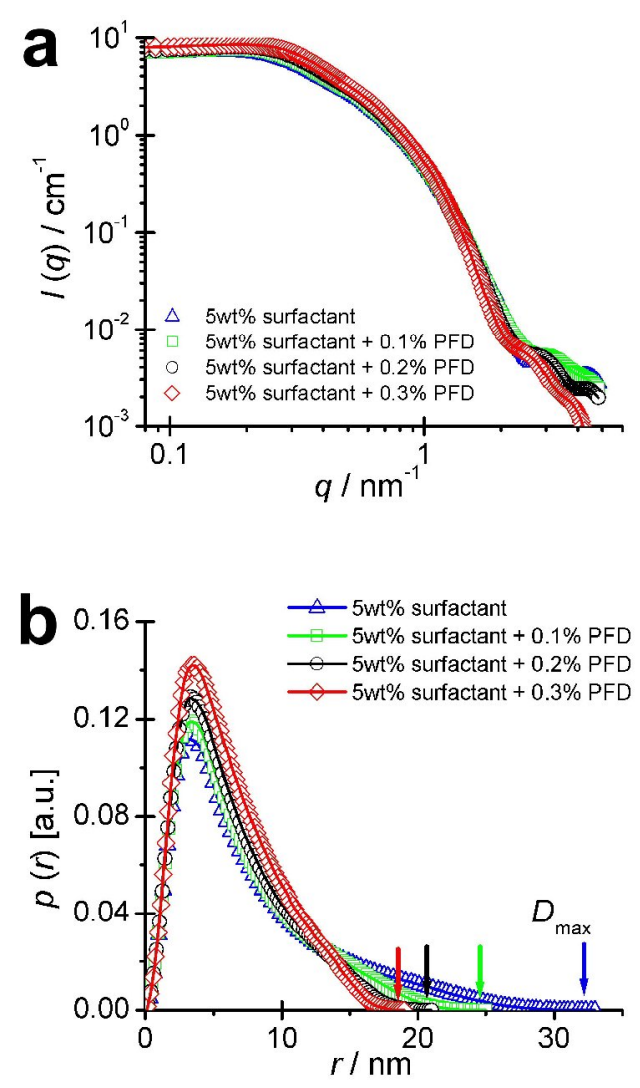

Figure 4: (a) The normalized scattering intensity $I(q)$, and (b) the corresponding pair-distance distribution functions (PDDF), $p(r)$, extracted by the GIFT analysis as a function of the PFD concentration obtained at $25^{\circ} \mathrm{C}$. 

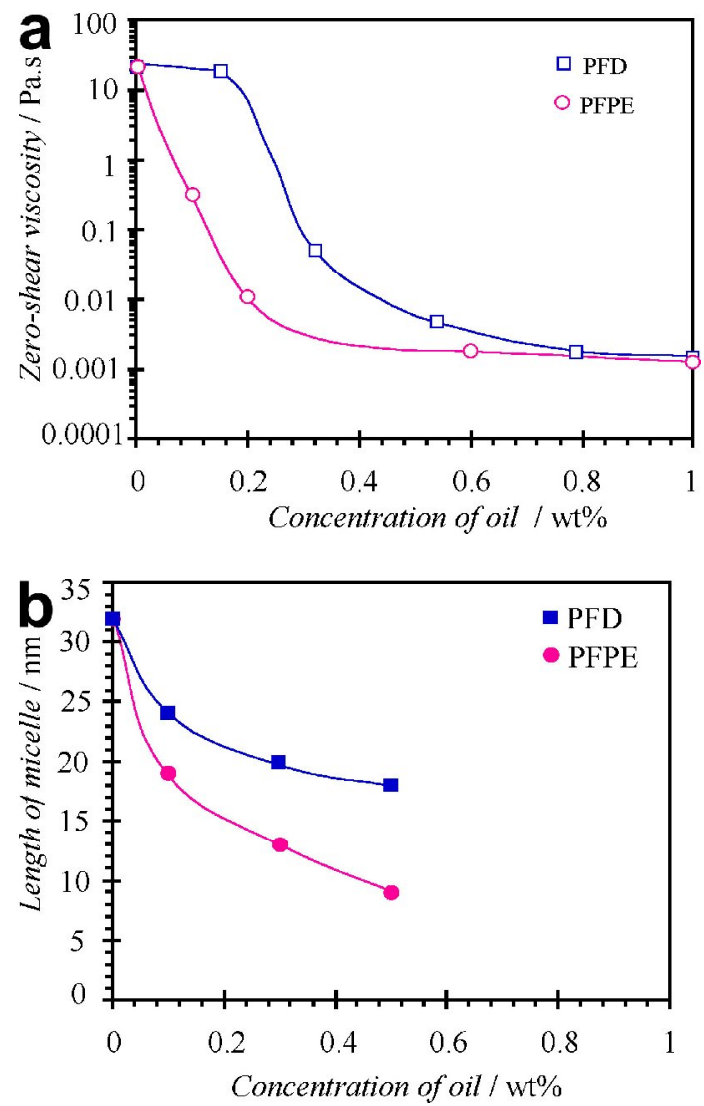

Figure 5: (a) Zero-shear viscosity as a function of oils concentration for the $5 \mathrm{wt} \%$ surfactant system at $25^{\circ} \mathrm{C}$, and (b) the corresponding length of micelles versus oils concentration at selected oil concentrations. 

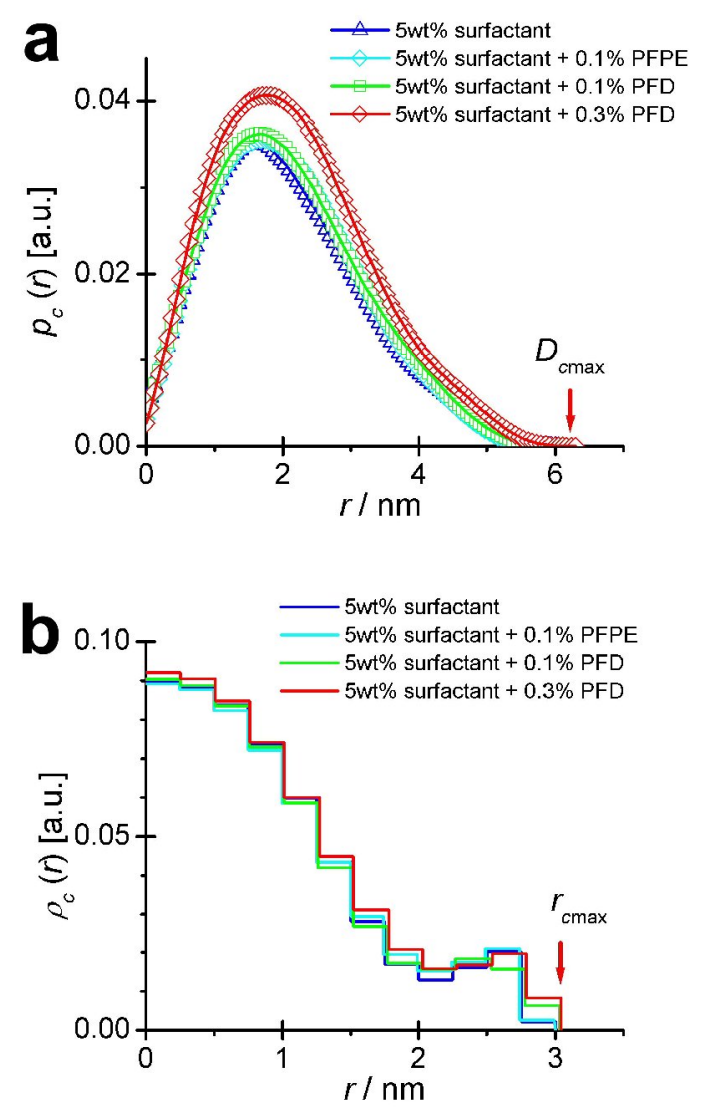

Figure 6: The model-free cross-section analysis. (a) The cross section PDDFs, $p_{\mathrm{c}}(r)$, for the $5 \mathrm{wt} \%$ surfactant/water systems at different concentrations of oils, and (b) the corresponding cross-section radial electron density profile $\Delta \rho_{\mathrm{c}}(r)$ deconvolution procedure. 

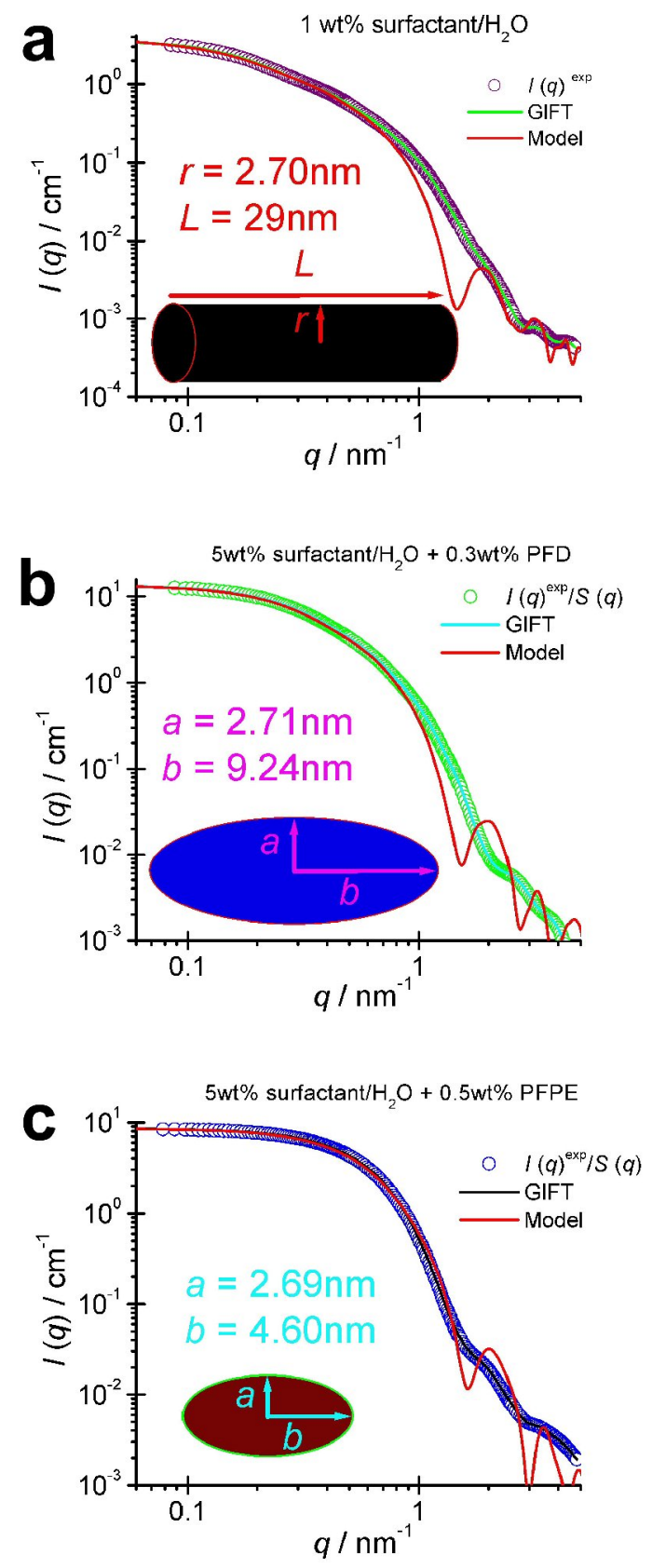

Figure 7: The model calculation and fit to the experimental X-ray scattered intensities of the (a) $1 \mathrm{wt} \%$ surfactant/water, (b) $5 \mathrm{wt} \%$ surfactant/water $+0.3 \mathrm{wt} \% \mathrm{PFD}$, and (c) $5 \mathrm{wt} \%$ surfactant/water $+0.5 \mathrm{wt} \%$ PFPE as typical examples. The data were fitted using model of a homogeneous cylinder and a homogeneous prolate ellipsoid. 